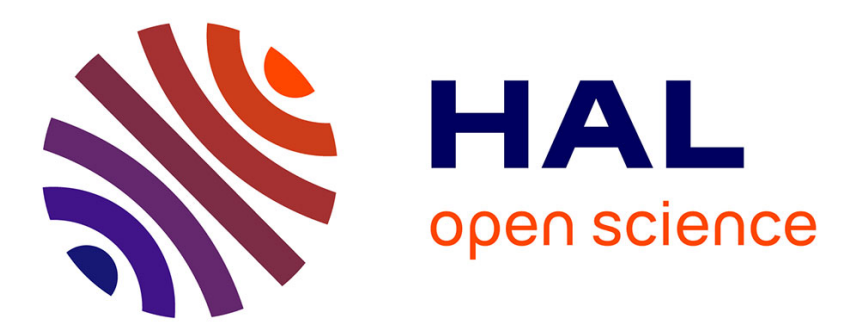

\title{
Effective properties of nonlinear laminated composites with perfect adhesion
}

\author{
Frédéric Lebon, Reinaldo Rodríguez-Ramos, J.C. Lopez-Realpozo, J. \\ Bravo-Castillero, J. Guinovart-Diaz, A. Mesejo
}

\section{- To cite this version:}

Frédéric Lebon, Reinaldo Rodríguez-Ramos, J.C. Lopez-Realpozo, J. Bravo-Castillero, J. GuinovartDiaz, et al.. Effective properties of nonlinear laminated composites with perfect adhesion. Journal of Applied Mechanics, 2006, 73 (1), pp.174-178. 10.1115/1.2061907 . hal-00089017

\section{HAL Id: hal-00089017 https://hal.science/hal-00089017}

Submitted on 7 Mar 2018

HAL is a multi-disciplinary open access archive for the deposit and dissemination of scientific research documents, whether they are published or not. The documents may come from teaching and research institutions in France or abroad, or from public or private research centers.
L'archive ouverte pluridisciplinaire HAL, est destinée au dépôt et à la diffusion de documents scientifiques de niveau recherche, publiés ou non, émanant des établissements d'enseignement et de recherche français ou étrangers, des laboratoires publics ou privés. 


\section{Effective Properties of Nonlinear Laminated Composites With Perfect Adhesion}

\section{F. Lebon ${ }^{1}$}

Laboratoire de Mécanique de d'Acoustique, Université Aix-Marseille 1, 31 Chemin Joseph-Aiguier, 13402 Marseille Cedex 20, France

e-mail: lebon@1ma.cnrs-mrs.fr

\section{R. Rodríguez-Ramos}

e-mail: reinaldo@matcom.uh.cu

\section{J. C. Lopez-Realpozo}

\section{J. Bravo-Castillero}

\section{R. Guinovart-Diaz}

\section{A. Mesejo}

Facultad de Matemática y Computación, Universidad de la Habana, San Lázaro y L, CP. 10400, Vedado, Habana, Cuba

This paper is devoted to the homogenization of a nonlinear onedimensional problem as a particular case of laminated composite and its solution by a Wavelet-Galerkin method. This approach is an extension of this method to nonlinear problems. Theoretical results are given and numerical examples are presented.

\section{Introduction}

Many problems of structural analysis are concerned with heterogeneous media. Theoretical approaches are necessary because of the impossibility to solve numerically real problems, even for a nonlinear behavior. In this paper we are interested in the computation of the effective properties for a periodic heterogeneous media in a special type one-dimensional no-linear elasticity. The following theory can be applied to laminated composites (see Fig. 1) usually used in civil engineering, aircrafts design and for certain applications to ultrasonic transducers. The idea is to consider the material as being a juxtaposition of identical cells, with identical geometrical and mechanical properties. So by multi-scale asymptotic expansion methods it is possible to obtain homogenized equations depending on nonlinear problems on the microstructure and on the global scale. These problems can be solved analytically in one dimension but it is necessary to introduce efficient solvers for two- or three-dimension problems. In the last decades many authors have tried to develop different numerical methods in order to solve the local and global problems, for instance the finite element method [1], Fourier transform [2], and wavelet transform [3]. In this paper a Wavelet-Galerkin method is introduced in order to solve the local and global nonlinear problems in one dimension. The advantages of this method are: (i) there is no creation of mesh contrary to the finite element method,

\footnotetext{
${ }^{1}$ Corresponding author
}

(ii) there is no Gibbs phenomena contrary to the Fourier transform, and (iii) the intrinsic adaptivity of wavelets.

The paper is organized as follows: in Sec. 2, we present the one-dimensional nonlinear elastic problem. Section 3 is devoted to the homogenization of the problem. Section 4 is devoted to the numerical solution by a Wavelet-Galerkin method (WaveletElement method). Finally, Sec. 5 contains some numerical examples with two or three types of materials.

\section{A One-Dimensional Nonlinear Problem}

Let us consider a nonhomogeneous thin bar of length $L$ under the action of volume forces $f(x)$. This bar is supposed to model a laminated composite where the length of each stripe is smaller than the perpendicular direction as shown in Fig. 1. The displacement problem of such a bar is given by the following onedimensional elliptic equation

$$
\frac{d}{d x} \mathcal{F}\left(x, \frac{d u}{d x}\right)+f(x)=0
$$

together with the boundary conditions

$$
\left.u\right|_{x=0}=u^{0},\left.\quad \mathcal{F}\left(x, \frac{d u}{d x}\right)\right|_{x=L}=S^{0}
$$

where $\mathcal{F}:[0, L] \times R \rightarrow R, f:[0, L] \rightarrow R$ and $u:[0, L] \rightarrow R$.

We assume that the bar is a composite material with periodic structure. For instance, the bar is composed of $p \cdot n$ smaller bars made of $p$ different homogeneous materials (see Fig. 2). The $p$ materials form a periodic cell of length $l$ and it is supposed that $l \ll L$ (equivalent to $n \gg 1$ ). Under these conditions, the relation between the stress and the strain, given by the map $\mathcal{F}$, is a periodic one in the spatial variable $x$, then

$$
\sigma(x)=\mathcal{F}\left(x, \frac{d u}{d x}\right)=\mathcal{F}^{\kappa}\left(x, \frac{d u}{d x}\right), \quad \kappa=1, \ldots, p
$$

for all $x$ in the $\kappa$ small bar of the periodic cell. We also suppose that the nonlinear constitutive relation (3) is invertible. Let $l_{\kappa}, \kappa$ $=1, \ldots, p$ be the length of the $\kappa t h$ bar in the periodic cell, then

$$
l=\sum_{\kappa=1}^{p} l_{\kappa}
$$

and together with the boundary conditions (2) we have the continuity conditions $[u]=0$ and $[\sigma]=0$ at the joints of the rods, i.e., at points $x_{i}^{\kappa}$. Here $x_{i}^{\kappa}=(i-1) \cdot l+\sum_{m=1}^{\kappa} l_{m}$ for $i=1, \ldots, n, \kappa=1, \ldots, p$ and $\left.[\cdot]\right|_{x=x_{i}^{\kappa}}$ means the difference between the left and right limits at the point $x_{i}^{\kappa}$. Equation (1) together with boundary conditions (2), the continuity and conjugation conditions form a typical problem of heterogeneous nonlinear elasticity theory.

\section{Homogenization of the Problem}

The homogenized equation corresponding to Eq. (1) can be derived by the method of multi-scale asymptotic expansions. In the present problem, there are two natural spatial length scales, one measuring variations within the periodic cell and the other measuring variations within the whole bar. For this reason, we introduce a new variable $\xi=x / \alpha$, where $\alpha$ is a small parameter related to the periodic structure of the material. The variable $\xi$ is called the fast variable in relation with $x$, which is the slow variable.

We define now the functions $\mathcal{F}^{\alpha}(x, \cdot), u^{\alpha}(x)$, and $\sigma^{\alpha}(x)$ by

$$
\mathcal{F}^{\alpha}(x, \cdot)=\mathcal{F}\left(\frac{x}{\alpha}, \cdot\right)=\mathcal{F}(\xi, \cdot), \quad u^{\alpha}(x)=u\left(\frac{x}{\alpha}\right)=u(\xi),
$$




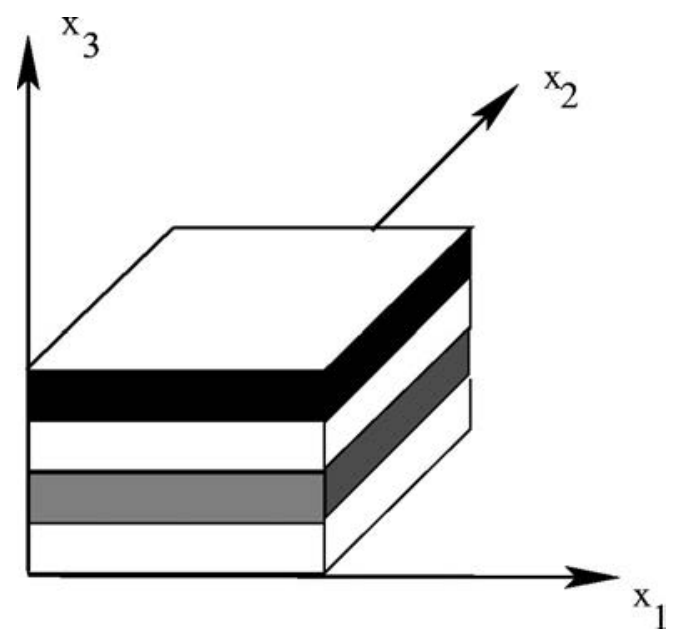

Fig. 1 A laminated composite: the length in directions $x_{1}$ and $x_{2}$ is small with respect to the length in direction $x_{3}$

$$
\sigma^{\alpha}(x)=\mathcal{F}^{\alpha}\left(x, \frac{d u}{d x}\right)
$$

and consider the problem

$$
\begin{gathered}
\frac{d}{d x} \mathcal{F}^{\alpha}\left(x, \frac{d u^{\alpha}}{d x}\right)+f^{\alpha}(x)=0 \\
\left.u^{\alpha}\right|_{x=0}=u^{0},\left.\quad \mathcal{F}^{\alpha}\left(x, \frac{d u^{\alpha}}{d x}\right)\right|_{x=\alpha L}=S^{0} \\
{\left.\left[u^{\alpha}\right]\right|_{x=\alpha x_{i}^{\kappa}}=0, \quad i=1, \ldots, n-1, \quad \kappa=1, \ldots, p,} \\
{\left.\left[u^{\alpha}\right]\right|_{x=\alpha x_{n}^{\kappa}}=0, \quad \kappa=1, \ldots, p-1} \\
{\left.\left[\sigma^{\alpha}\right]\right|_{x=\alpha x_{i}^{\kappa}}=0, \quad i=1, \ldots, n-1, \quad \kappa=1, \ldots, p,} \\
{\left.\left[\sigma^{\alpha}\right]\right|_{x=\alpha x_{n}^{\kappa}=0, \quad \kappa=1, \ldots, p-1}}
\end{gathered}
$$

where $f^{\alpha}(x)=f(x / \alpha)$. The parameter $\alpha$ is properly chosen in order to make the function $\mathcal{F}^{\alpha}$ 1-periodic in $\xi$. We search a solution of Eqs. (6)-(9) with the aid of the following two-scale asymptotic expansion of the unknown function $u^{\alpha}$

$$
u^{\alpha}(x)=v(x)+\alpha u_{1}(x, \xi)+\alpha^{2} u_{2}(x, \xi)+\alpha^{3} u_{3}(x, \xi)+\ldots
$$

This means that the total dependence on $x$ is obtained directly and through the variable $\xi$. For an explanation of the physical meaning of the expansion (10) and some other interesting considerations see Ref. [4]. We substitute Eq. (10) into Eq. (6), use the chain rule and make an expansion of $\mathcal{F}^{\alpha}$ in a Taylor series [4]. After some manipulations equations for orders $-1,0$, and 1 are obtained. Finally, we obtain local problems [4,5] (LP) for the calculation of the functions $v(x)$ and $u_{i}(x, \xi), i \geqslant 1$. For simplicity, we only deal with the problems for the calculation of the functions $v(x)$ and $u_{1}(x, \xi)$. Suppose there exists a 1-periodic solution $u_{1}(x, \xi)$ of the following nonlinear local problem (LP0) where $v^{\prime}(x)$ is treated as an independent parameter.

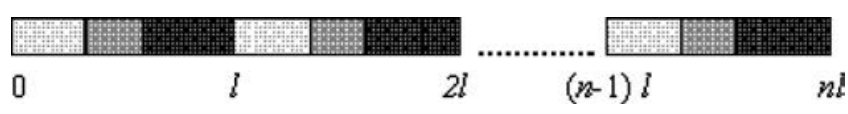

Fig. 2 The periodic structure of the heterogeneous bar for $p$ $=3$
LP0:

$$
\begin{gathered}
\frac{\partial}{\partial \xi} \mathcal{F}^{\alpha}\left(x, v^{\prime}(x)+\frac{\partial u_{1}}{\partial \xi}(x, \xi)\right)=0 \\
u_{1}(x, 0)=0 \\
{\left.\left.\left[u_{1}(x, \xi)\right]\right|_{x=x_{i}^{\kappa}=0, \quad 1 \leqslant i \leqslant p-1, \quad 1 \leqslant \kappa \leqslant p}\left[u_{1}(x, \xi)\right]\right|_{x=x_{n}^{\kappa}}=0, \quad 1 \leqslant \kappa \leqslant p-1} \\
{\left.\left[\mathcal{F}^{\alpha}\left(x, v^{\prime}(x)+\frac{\partial u_{1}}{\partial \xi}(x, \xi)\right)\right]\right|_{x=x_{i}^{\kappa}}=0, \quad 1 \leqslant i \leqslant p-1,1 \leqslant \kappa \leqslant p} \\
{\left.\left[\mathcal{F}^{\alpha}\left(x, v^{\prime}(x)+\frac{\partial u_{1}}{\partial \xi}(x, \xi)\right)\right]\right|_{x=x_{n}^{\kappa}}=0, \quad 1 \leqslant \kappa \leqslant p-1}
\end{gathered}
$$

It follows that $u_{1}$ depends on $x$ through $v^{\prime}(x)$, in other words $u_{1}=u_{1}\left(v^{\prime}(x), \xi\right)$. Once $u_{1}$ is known the solution of the recurrent sequence problems are provided $u_{2}(x, \xi), u_{3}(x, \xi), \ldots$. Since $u_{1}$ $=u_{1}\left(v^{\prime}(x), \xi\right)$ we can write

$$
\frac{d}{d x} \hat{\mathcal{F}}^{\alpha}\left(v^{\prime}(x)\right)-\hat{f}^{\alpha}(x)=0
$$

where

$$
\begin{gathered}
\hat{\mathcal{F}}^{\alpha}\left(v^{\prime}(x)\right):=\left\langle\mathcal{F}^{\alpha}\left(x, v^{\prime}(x)+\frac{\partial u_{i}}{\partial \xi}(x, \xi)\right)\right\rangle, \\
\hat{f}^{\alpha}(x):=\left\langle f^{\alpha}(x)\right\rangle \text { and }\langle\cdot\rangle:=\int_{0}^{1} \cdot d \xi
\end{gathered}
$$

The last integral is called the one-dimensional average operator. By definition $v(x)$ is a continuous function, then the continuity conditions are obviously satisfied. The averaged problem (AP) of the original problems (1) and (2) is AP

$$
\frac{d}{d x} \hat{\mathcal{F}}\left(v^{\prime}(x)\right)-\hat{f}^{\alpha}(x)=0
$$

$$
v(0)=u^{0},\left.\quad \hat{\mathcal{F}}\left(v^{\prime}(x)\right)\right|_{x=L}=S^{0}
$$

where we define

$$
\hat{\mathcal{F}}(y):=\left\langle\mathcal{F}\left(\xi, y+\frac{\partial \theta}{\partial \xi}(\xi, y)\right)\right\rangle
$$

As a consequence of LPO we have

$$
\hat{\mathcal{F}}^{\alpha}\left(v^{\prime}(x)\right)=\mathcal{F}^{\alpha}\left(x, v^{\prime}(x)+\frac{\partial u_{1}}{\partial \xi}(x, \xi)\right)
$$

Thus what we need for the construction of the averaged equation (17) is to solve problem LP0 and to use Eq. (20) for stating $\hat{\mathcal{F}}^{\alpha}\left(v^{\prime}(x)\right)$. The normal approach is as follows. We consider the following parametric problem (PP) depending on parameter $y$ $\operatorname{PP0}(y)$ :

$$
\begin{gathered}
\frac{\partial}{\partial \xi} \mathcal{F}\left(\xi, y+\frac{\partial \theta}{\partial \xi}(\xi, y)\right)=0 \\
\theta(0, y)=0 \\
{\left.[\theta(\xi, y)]\right|_{\xi=\xi_{i}^{\kappa}=0, \quad 1 \leqslant i \leqslant p-1, \quad 1 \leqslant \kappa \leqslant p,}} \\
{\left.[\theta(\xi, y)]\right|_{\xi=\xi_{n}^{\kappa}=0, \quad 1 \leqslant \kappa \leqslant p-1}}
\end{gathered}
$$




$$
\begin{gathered}
{\left.\left[\mathcal{F}\left(\xi, y+\frac{\partial \theta}{\partial \xi}(\xi, y)\right)\right]\right|_{\xi=\xi_{i}^{\kappa}}=0, \quad 1 \leqslant i \leqslant p-1, \quad 1 \leqslant \kappa \leqslant p} \\
{\left.\left[\mathcal{F}\left(\xi, y+\frac{\partial \theta}{\partial \xi}(\xi, y)\right)\right]\right|_{\xi=\xi_{n}^{\kappa}}=0, \quad 1 \leqslant \kappa \leqslant p-1}
\end{gathered}
$$

From the definitions (5) and from the statement of the problem LP0 it is clear that the solution $u_{1}$ of LP0 satisfies

$$
u_{1}(x, \xi)=\theta\left(\xi, v^{\prime}(x)\right)
$$

where $\theta(\xi, y)$ is the solution of $\operatorname{PP} 0(y)$. Therefore, we solve $\mathrm{PP} 0(y)$ for the construction of the averaged equation and define $\hat{\mathcal{F}}(y)$ from Eq. (19).

\section{Numerical Solution by the Wavelet-Element Method}

4.1 General Algorithms. As proposed in former papers [3,6], to solve problems PP0 and AP we use the compactly supported wavelets introduced by Daubechies [7] and particularly the Haar basis. These bases are classical bases of $L^{2}(\mathbb{R})$ and are defined from the data of a function $\varphi$ called the scaled function and a function $\psi$, orthogonal to the first one called the associated wavelet. In this paper, we have chosen the classical Haar basis. In the following the unknowns of problems PP0 and AP are approximated in a finite space $V_{J}$ ( $J$ be given). A function $f$ is decomposed in this space on the form

$$
f=\sum_{k \in \Lambda_{0}} f_{0 k} \phi_{0 k}+\sum_{j=0}^{j=J} \sum_{k \in \Lambda_{j}} f_{j k} \psi_{j k}
$$

where $\Lambda_{j}=\left[0,2^{j}-1\right]$. The local problem $\operatorname{PP} 0(y)$ takes on a vectorial form (discretized nonlinear system)

$\operatorname{NLS} 0(y)$ : Find $\bar{\theta}_{J}$ such that $G_{y}\left(\bar{\theta}_{J}\right)=0$

$\bar{\theta}_{J}=\left(\theta_{j k}\right)_{j_{0} \leqslant j \leqslant J, k \in \Lambda_{j}}$ is the vector built from the wavelet coefficients of $\theta . G_{y}\left(\bar{\theta}_{J}\right)$ is a nonlinear vectorial function obtained from the discretization of the nonlinear operator $\mathcal{F}^{\alpha}$. The solution of problem NLSO( $y$ ) is obtained using the generalized Newton method (GNM) which is an extension of the classical Newton method to continuous nondifferentiable equations [8]. If $\mathcal{F}^{\alpha}$ is differentiable this method is reduced to Newton's method.

$$
\bar{\theta}_{J}^{0} \text { given, } \partial G_{y}\left(\bar{\theta}_{J}^{i}\right)\left(\bar{\theta}_{J}^{i+1}-\bar{\theta}_{J}^{i}\right)=-G_{y}\left(\bar{\theta}_{J}^{i}\right)
$$

$\partial G_{y}\left(\bar{\theta}_{J}^{i}\right)$ is a matrix in the set of the generalized Jacobian of $G_{y}$ at $\bar{\theta}_{J}^{i}$. The initial global problem AP can be written as a problem of minimization under the constraint $v(0)=u_{0}$ of the potential energy of the system $\phi(w)=\frac{1}{2} \int_{0}^{L} \hat{\mathcal{F}}\left(w^{\prime}(x)\right) w^{\prime}(x) d x-\int_{0}^{L} f w d x$. In order to solve this problem, we have chosen to use the Lagrangian method. This method consists in introducing a new variable $\lambda$, called the Lagrange multiplier, to treat the constraint $v(0)=u_{0}$. We build a function called Lagrangian $L(w, \mu)=\phi(w)+\mu\left(w(0)-u_{0}\right)$ such that the solution is a saddle point of the Lagrangian (minimum for the displacement, maximum for the multiplier). This method could be improved by the introduction of the augmented Lagrangian method which consists in adding a new term depending on the penalty factor $r$. The augmented Lagrangian is $L_{r}(w, \mu)=\phi(w)$ $+\mu\left(w(0)-u_{0}\right)+(r / 2)\left(w(0)-u_{0}\right)^{2}$. To find the saddle point solution, we write that the derivative of the augmented Lagrangian is equal to zero. In other words, we have $\nabla L_{r}(v, \lambda)(w, \mu)=0$. This problem is first discretized on a wavelet basis in the same way as in the previous part of this section (nonlinear discretized averaged problem):

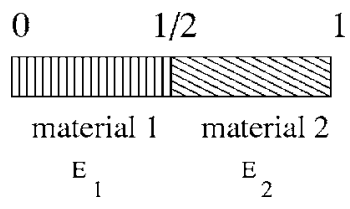

Fig. 3 The first example with two materials on each microstructure

$N L A P:$ Find $\left(\bar{v}, \bar{\lambda}_{J}\right)$ such that

$$
\hat{G}\left(\bar{v}_{J}, \bar{\lambda}_{J}\right)=0
$$

This discretized problem is solved by the Generalized Newton method.

4.2 Application to an Illustrating Example. Assume that the function $\mathcal{F}$ is given by

$$
\mathcal{F}\left(x, u^{\prime}\right):= \begin{cases}E(x) u^{\prime} & \text { if } u^{\prime}<\varepsilon_{s}(x) \\ \hat{E}(x) u^{\prime}+(E(x)-\hat{E}(x)) \varepsilon_{s}(x) & \text { if } u^{\prime} \geqslant \varepsilon_{s}(x)\end{cases}
$$

and that for all $x$ in the interval $\left[x_{i}^{\kappa}, x_{i}^{\kappa+1}\right](1 \leqslant i \leqslant N, 1 \leqslant \kappa \leqslant p$, $\left.x_{1}^{1}=0, x_{i}^{p+1}=x_{i+1}^{1}, x_{N}^{p+1}=L\right)$ occupied by the $\kappa$ th small periodic rod, we have $E(x)=E_{\kappa}, \hat{E}(x)=\hat{E}_{\kappa}, \varepsilon_{s}(x)=\varepsilon_{s}^{\kappa}\left(E_{\kappa}, \hat{E}_{\kappa}, \varepsilon_{s}^{\kappa}\right.$ be given). In this case, it is possible to compute the elementary terms of the tangent matrix and of the right-hand side necessary for the generalized Newton method

$$
\begin{gathered}
{\left[\partial G_{y}(.)\right]_{K K^{\prime}}=\sum_{\kappa=1}^{\kappa=p} E_{\kappa}^{a} \int_{\xi^{\kappa}}^{\xi^{\kappa+1}} \Psi_{j k} \Psi_{j^{\prime} k^{\prime}} d \xi} \\
{\left[G_{y}(.)\right]_{K}=-\sum_{\kappa=1}^{\kappa=p} E_{\kappa}^{a} y \int_{\xi^{\kappa}}^{\xi^{\kappa+1}} \Psi_{j k} d \xi+\sum_{\kappa=1}^{\kappa=p}\left(E_{\kappa}^{b}-E_{\kappa}^{c}\right) \epsilon_{\kappa}^{s} \int_{\xi^{\kappa}}^{\xi^{\kappa+1}} \Psi_{j k} d \xi}
\end{gathered}
$$

with $K=k+1+\sum_{i=0}^{i=j-1} 2^{i}, E_{\kappa}^{a}=E_{\kappa}$ or $\hat{E}_{\kappa}$. If $E_{\kappa}^{a}=E_{\kappa}$ then $E_{\kappa}^{b}=E_{\kappa}^{c}=E_{\kappa}$, and if $E_{\kappa}^{a}=\hat{E}_{\kappa}$ then $E_{\kappa}^{b}=\hat{E}_{\kappa}$ and $E_{\kappa}^{c}=E_{\kappa}$. In the next paragraphs, the deformation is decomposed on the Haar basis. Due to the antiperiodicity of the deformation and to the periodicity of the displacement, we have

$$
\frac{\partial \theta}{\partial \xi}(\xi)=\sum_{j=0}^{j=J} \sum_{k \in \Lambda_{j}} \theta_{j k} \Psi_{j k}, \quad \theta(\xi)=\sum_{j=0}^{j=J} \sum_{k \in \Lambda_{j}} \theta_{j k} \bar{\Psi}_{j k}
$$

where $\bar{\Psi}$ is the primitive of $\Psi$, i.e., the Schauder basis.

\section{Numerical Examples}

5.1 First Example (Local Problem). In this first example, we consider a two-phase composite. The elementary cell is split in two equal parts. We denote with indices $i=1$ or 2 the coefficients corresponding to each material (Fig. 3). The first step is to compute the terms of the tangent matrix. $E(y)$ is constant on each compact support of each wavelet $\Psi_{j k}$. The orthogonality of the Haar basis leads to simple values

$$
\begin{gathered}
{\left[\partial G_{y}(.)\right]_{11}=\frac{1}{2}\left(E_{1}^{a}+E_{2}^{a}\right), \quad\left[\partial G_{y}(.)\right]_{K K}=E_{k}^{a}, \quad K \geqslant 2,} \\
{\left[\partial G_{y}(.)\right]_{K K^{\prime}}=0, \quad K \neq K^{\prime}}
\end{gathered}
$$

The term $\left[\partial G_{y}(.)\right]_{11}$ corresponds to the average of the Young modulus over the cell, i.e., on the compact support of the wavelet. The terms $\left[\partial G_{y}(.)\right]_{K K}$ correspond to the average of the Young modulus on the compact support of each wavelet $\Psi_{j k}$. The diagonality of the matrix shows the independence between each level. Due to the same arguments in the previous paragraph, only the 


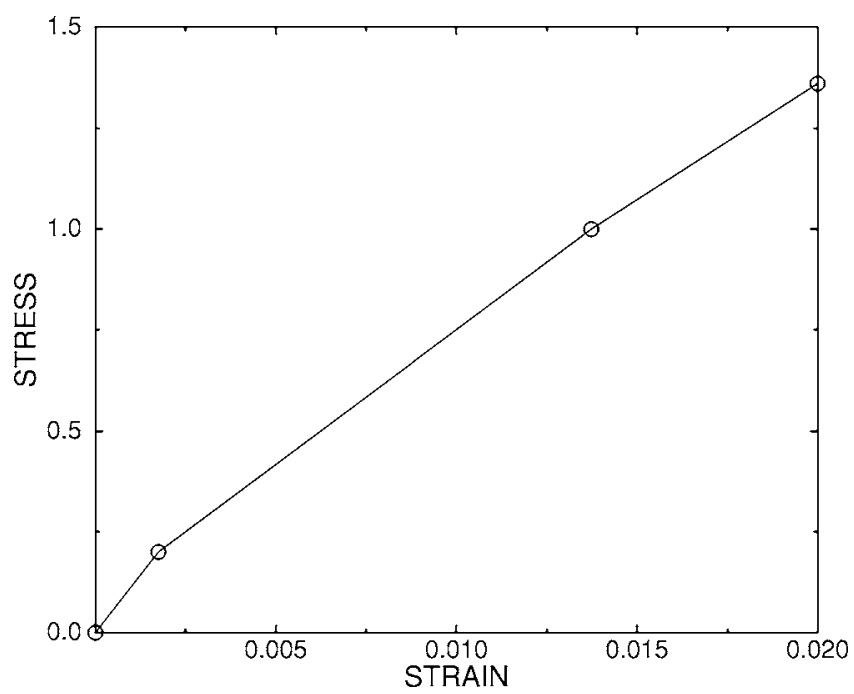

Fig. 4 Macroscopic behavior law (example with two materials)

first component of the right-hand side of (27) has a value not equal to zero. We obtain three different possible values. Thus

$$
\begin{gathered}
{\left[G_{y}(.)\right]_{1}=\frac{1}{2}\left(E_{2}^{a}-E_{1}^{a}\right) y+\frac{1}{2}\left(E_{1}^{b}-E_{1}^{c}\right) \epsilon_{1}^{s}+\frac{1}{2}\left(E_{2}^{b}-E_{2}^{c}\right) \epsilon_{2}^{s}} \\
{\left[G_{y}(.)\right]_{K}=0 \text { if } K>1}
\end{gathered}
$$

The global tangent matrix is diagonal and the right-hand side has only its first term which is not equal to zero. We present a numerical application with $E_{1}=80 \mathrm{GPa}, E_{2}=200 \mathrm{GPa}, \hat{E}_{1}$ $=40 \mathrm{GPa}, \hat{E}_{2}=100 \mathrm{GPa}, \sigma_{1}^{s}=0.2 \mathrm{GPa}$, and $\sigma_{2}^{s}=1 \mathrm{GPa}$. The macroscopic deformation $y$ increases from 0 to $0.6 \%$. Due to the form of the constitutive equation, the generalized Newton method converges in one or two iterations. At each iteration this method is initialized with the solution at the last step and so, if the solution remains in the same region of linearity, the convergence is obtained in only one iteration. If the region of linearity is changed, due to the evolution of $y$, the convergence is obtained in two iterations. Figure 4 shows the macroscopic response, i.e., a three phase nonlinear elasticity, these values coincide with the results presented in Refs. [4,5].

5.2 Second Example. The second example concerns a threephase material. We denote with indices $i=1,2,3$ the coefficients of each material (Fig. 5). Due to the form of the microstructure, the problem is more complex than the former one. The matrix is not a diagonal one. There are interactions between the two first levels of approximation. Thus, using the same notations, we obtain the following tangent matrix

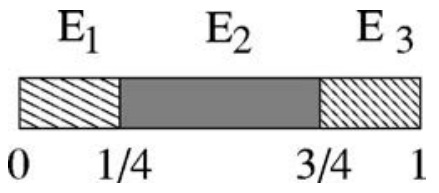

Fig. 5 The second example: a composite with three materials on each microstructure

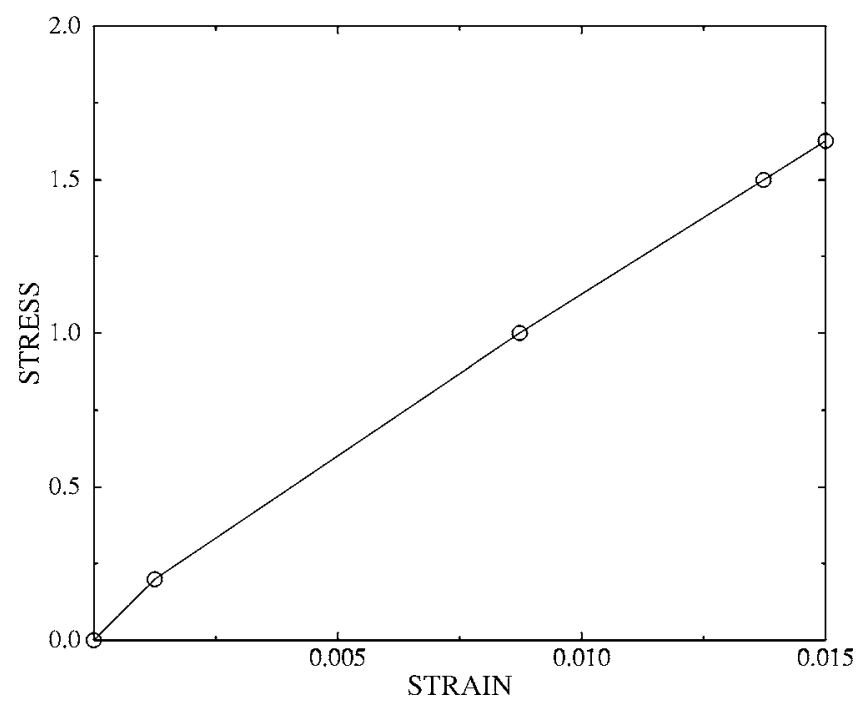

Fig. 6 Macroscopic behavior law (example with three materials)

$\left[\partial G_{y}().\right]$

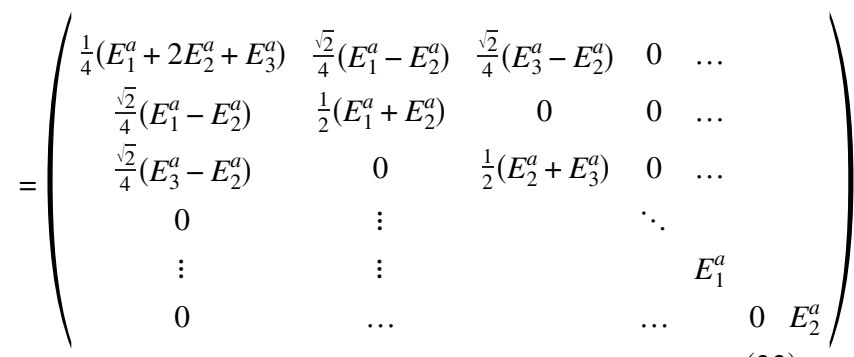

The right-hand side has only its three first terms which are not equal to zero, thus in this case only three coefficients are not equal to zero $\theta_{00}, \theta_{10}$, and $\theta_{11}$. We present a numerical application with $E_{1}=80 \mathrm{GPa}, \quad E_{2}=200 \mathrm{GPa}, \quad E_{3}=400 \mathrm{GPa}, \quad \hat{E}_{1}=40 \mathrm{GPa}, \quad \hat{E}_{2}$ $=100 \mathrm{GPa}, \quad \hat{E}_{3}=200 \mathrm{GPa}, \quad \sigma_{1}^{s}=0.2 \mathrm{GPa}, \sigma_{2}^{s}=1 \mathrm{GPa}$, and $\sigma_{3}^{s}$ $=1.5 \mathrm{GPa}$. Figure 6 shows the results obtained for the global response: a nonlinear elasticity law with four phases, these values are the same as those in the analytical approach presented in Refs. $[4,5]$.

\section{Conclusion}

In this paper, we have presented results concerning the computations of the effective properties of laminated composites. Numerical results show that the wavelet element method is a powerful tool for the characterization of composite materials. In the future, we intend to extend the methods to more general laminated composite (noninvolved behavior, non-perfect interfaces).

\section{Acknowledgment}

The work was completed while the author RRR was visiting the Laboratoire de Modélisation en Mécanique from Université Pierre et Marie Curie, Paris supported by the CNRS. The support of project PNCB IBMFQ 09-2004 is also acknowledged. The authors thank Professor G.A. Maugin for his helpful comments. 


\section{References}

[1] Léné, F., 1984, "Contribution L'étude des Matériaux Composites et a leur Endommagement," Ph.D. thesis, Pierre et Marie Curie University.

[2] Moulinec, H., and Suquet, P., 1994, "A Fast Numerical Method for Computing the Linear and Non Linear Mechanical Properties of Composites," C. R. Acad. Sci. III, 318, pp. 1417-1423.

[3] Dumont, S., and Lebon, F., 1996, "Representation of Plane Elastostatics Operators in Daubechies Wavelets," Comput. Struct., 60, pp. 561-569.
[4] Pobedria B. E., 1984, Mechanics of Composite Materials, 1st ed., Moscow State University Press, Moscow (in Russian).

[5] Bachvalov, N. S., and Panasenko, G. P., 1989, Homogenization of Processes in Periodic Media, Kluwer, Dordrecht.

[6] Dumont, S., and Lebon, F., 1996, "Wavelet-Galerkin Method for Heterogeneous Media," Comput. Struct., 61, pp. 55-65.

[7] Daubechies, I., 1992, "Orthonormal Bases of Compactly Supported Wavelets," Commun. Pure Appl. Math., 41, pp. 909-998.

[8] Alart, P., and Lebon, F., 1995, "Solution of Frictional Contact Problems Using ILU and Coarse/Fine Preconditioners,” Comput. Mech., 16, pp. 98-105. 